\title{
NeuroImage
}

ELSEVIER

www.elsevier.com/locate/ynimg

NeuroImage 32 (2006) $778-789$

\section{Magnetoencephalography (MEG) determined temporal modulation of visual and auditory sensory processing in the context of classical conditioning to faces}

\author{
R.J. Dolan, ${ }^{a, *}$ H.J. Heinze, ${ }^{b}$ R. Hurlemann, ${ }^{b}$ and H. Hinrichs ${ }^{b}$ \\ ${ }^{a}$ Institute of Neurology, University College London, UK \\ ${ }^{\mathrm{b}}$ Clinic of Neurology II, University of Magdeburg, Germany
}

Received 7 November 2005; revised 7 March 2006; accepted 5 April 2006

Available online 19 June 2006

\begin{abstract}
Using magnetoencephalography (MEG), we determined the time course of sensory-evoked modulations during differential aversive conditioning to faces, with an aversive noise event (UCS). Conditioning was associated with the development of a differential event-related waveform peaking at approximately $150 \mathrm{~ms}$. Source analysis indicated the localization of this modulation to ventral occipital regions. In the auditory domain, a modulation of auditory-evoked responses to a probe sound was evident in a late component emerging at approximately $180 \mathrm{~ms}$ over sensors in fronto-temporal regions. The findings indicate the time course in processing sensory stimuli can be altered on the basis of their acquired value.

(C) 2006 Elsevier Inc. All rights reserved.
\end{abstract}

Keywords: MEG; Aversive conditioning; Face processing; Auditory processing

\section{Introduction}

Humans have exceptional abilities to recognize not only the identity of a face but also its more subtle aspects including emotional expression as well as the intentionality implicit in an expression. The former ability depends upon neural processes that involve occipito-temporal cortices, evidenced in the fact that damage to these regions results in prosopagnosia, a deficit in familiar face recognition (Meadows, 1974; Damasio et al., 1982). In normal healthy subjects, face-related activations in these regions are seen with functional neuroimaging that show a strong affiliation with activity in fusiform gyrus and in the lateral occipital complex (LOC) (Sergent et al., 1994; Dolan et al., 1996; Haxby et al., 1996; Kanwisher et al., 1997; Haxby et al., 2000). By contrast, an ability to make appropriate emotional judgments in response to

\footnotetext{
* Corresponding author.

E-mail address: r.dolan@fil.ion.ucl.ac.uk (R.J. Dolan).

Available online on ScienceDirect (www.sciencedirect.com).
}

faces depends upon other brain regions including the amygdala and superior temporal sulcus (STS) (Dolan, 2002; Winston et al., 2003).

The temporal dynamics in neuronal response to face stimuli have been widely studied using electrophysiological techniques including electroencephalography (EEG), magnetoencephalography (MEG), and direct intracranial recordings. These approaches highlight face-related neurophysiological responses that occur at approximately $200 \mathrm{~ms}$ post-stimulus presentation. Thus, facespecific electrophysiological responses have been recorded using scalp electrodes (Jeffreys, 1989; Bentin et al., 1996) and MEG (Lu et al., 1991; Sams et al., 1997; Watanabe et al., 2001). Direct recording from patients undergoing evaluation for surgery indicates that face responsive profiles with a similar time course can be recorded from fusiform gyrus, as well as middle occipital and temporal gyri (Allison et al., 1999, McCarthy et al., 1999). A functional specificity in terms of face detection and face identification has recently been proposed to account for regionally distinct visual-evoked occipito-temporal face responses (Liu et al., 2002).

Although characteristic electrophysiological face responses are seen at about $170-200 \mathrm{~ms}$ post-stimulus presentation, discriminatory responses to emotional faces are reported to occur as early as 100 to $120 \mathrm{~ms}$ post-stimulus (Halgren et al., 2000; Eimer and Holmes, 2002). The importance of stimulus value in influencing face processing is reinforced by a finding that intermodal binding for presentation of anger in voice and face elicits a EEG potential as early as $100 \mathrm{~ms}$ (Pourtois et al., 2000). Short-latency responses to aversive stimulus presentation are also seen in ventral prefrontal cortex using direct intracerebral recordings as early as $100 \mathrm{~ms}$ poststimulus presentation (Kawasaki et al., 2001). Thus, electrophysiological evidence indicates that faces with emotional value engage distinct processes, relative to non-emotional faces, at early time points following stimulus presentation.

In real life situations emotional responses to sensory stimuli, including faces, are often acquired through experience. One means 
to study emotional learning involves the use of classical conditioning paradigms. In classical conditioning, a previously neutral stimulus $(\mathrm{CS}+)$ acquires behavioral relevance by virtue of its ability to predict the future occurrence of an aversive event (UCS). An extensive animal and human literature indicates that this from of learning depends upon the amygdala (LeDoux, 1993; LaBar et al., 1998; Buchel and Dolan, 2000). In functional neuroimaging experiments of classical conditioning, as in studies of emotional face processing, it is widely reported that, in addition to amygdala activation, there are also CS + specific activations in sensory processing regions, which in the case of face stimuli includes enhanced fusiform and extrastriate cortical responses (Buchel and Dolan, 2000; Dolan, 2002).

In this study, we used the high temporal resolution offered by MEG to characterize the temporal dynamics within sensory cortices associated with acquisition of discriminatory responses to faces during classical conditioning. In this regard, MEG recordings have intrinsic advantages over EEG because for physical reasons the MEG has an improved spatial resolution (see for instance Chuang et al., 1995; Leahy et al., 1998). We used faces as CS stimuli and an acoustic burst of white noise as the UCS to address two distinct questions related to the sensory consequences of acquired emotional responses. Firstly, we wished to determine whether the temporal profile of face elicited electrophysiological responses show modulation as a function of a face acquiring predictive emotional value (i.e., differential CS+ versus CS- effects). Secondly, we wished to determine whether acquisition of discriminatory emotional responses were associated with modulation of sensory-evoked responses associated with expectation of UCS receipt.

\section{Materials and methods}

\section{Subjects}

Thirteen healthy right-handed female volunteers (age range $20-28$ years, mean 24.5 years, recruited by advertisement) with no past history of neurological or psychiatric disorders that might affect brain function participated in this MEG study. Two of them repeated the experiment because they had not appropriately responded to the stimuli due to a misunderstanding of the task. Another three subjects were excluded from the analysis because of movements during recording (as checked by an infrared video monitoring system). Consequently, ten subjects were included in the final MEG analysis. Eleven subjects were included in a separate behavioral study in order to demonstrate that the paradigm was sufficiently robust to ensure effective conditioning. Written informed consent was obtained before the experiment which was approved by a local ethics board.

\section{Stimuli and experimental design}

As visual stimuli, we selected two equally angry male faces (gray scale and in frontal view) from the Ekman series (Ekman and Friesen, 1982). Both faces were presented in a randomized order, with a stimulus duration of $800 \mathrm{~ms}$ and an inter stimulus interval (ISI) of $1700 \mathrm{~ms}$. Alternating across subjects, one of the angry faces (CS) was conditioned with an aversive burst (average intensity $100 \mathrm{~dB}$, binaural) of white noise (UCS) and consequently became the CS+. The UCS onset was $600 \mathrm{~ms}$ after the CS onset with the CS and UCS co-terminating at $800 \mathrm{~ms}$. The second face was not associated with the UCS and consequently became CS(CS- control condition). We employed a $50 \%$ partial reinforcement strategy in which one half of the $\mathrm{CS}+$ presentations were paired with the UCS $\left(\mathrm{CS}+{ }_{\text {paired }}\right.$ condition), whereas the other half were not $\left(\mathrm{CS}+_{\text {unpaired }}\right.$ condition). In order to assess how auditoryevoked responses were modulated by the $\mathrm{CS}+{ }_{\text {unpaired }}$ compared to $\mathrm{CS}-$, a single probe (auditory click, average intensity $80 \mathrm{~dB}$ ) was delivered in all trials at $600 \mathrm{~ms}$ after face onset. This corresponded to the expected delivery time of the noise UCS (see Fig. 1).

Before the start of the MEG recording, the UCS intensity was adjusted to each subject's aversive threshold (estimated by self report). To control for attentional factors, subjects were required to make push button responses to each visual stimulus throughout the experiment. This allowed us to use reaction times (RT) as a behavioral measure of fear conditioning (Critchley et al., 2002; Thiel et al., 2002). We hypothesized that expectation of an aversive UCS, associated with the CS+, would influence the subject's behavioral responses, resulting in shorter reaction times (our behavioral index of conditioning). We relied upon measurements of RTs rather than on skin conductance responses (SCRs) because the lengthy time constant of SCRs would not be compatible with the rapid sequence of stimuli applied in this study.

In total, we presented 800 stimuli over a period of $36 \mathrm{~min} .200$ stimuli were $\mathrm{CS}+$ paired with noise $\left(\mathrm{CS}+{ }_{\text {paired }}\right.$ condition), another 200 stimuli were $\mathrm{CS}+$ not paired $\left(\mathrm{CS}+{ }_{\text {unpaired }}\right.$ condition), and 400 stimuli were $\mathrm{CS}-(\mathrm{CS}-$ control condition), resulting in a balanced randomized presentation of the $\mathrm{CS}+$ and $\mathrm{CS}-$ face stimuli. The computer-generated auditory stimuli were delivered through MEG compatible plastic tubes, sealed by ear inserts, delivered to both ears. The visual stimuli, consisting of angry faces, were presented via computer-controlled back projection at a viewing distance of $120 \mathrm{~cm}$ and a visual angle of $9^{\circ}$ (horizontal) $\times 12^{\circ}$ (vertical). Throughout the experiment, a fixation point was present in the center of the screen (black background) in order to support subjects in minimizing eye blinks and eye movements. Subjects were familiarized with all stimuli during a 2-min preconditioning session.

\section{Recording and analysis}

Magnetoencephalography (MEG) signals were recorded using a 148-channel Magnes $2500 \mathrm{WH}$ whole-head MEG device equipped with magnetometer sensors (4DNeuroimaging Inc., San Diego). In addition, electrooculogram (EOG) signals were acquired using a Synamps amplifier (Neuroscan Inc., Herndon, VA) connected to an electrode behind the right lateral orbital angle (horizontal EOG) and an electrode below the right orbital limb (vertical EOG), both referenced to the left mastoid. The signals were filtered with a band pass of DC to $50 \mathrm{~Hz}$ and digitized with a sampling rate of $254 \mathrm{~Hz}$. Before further processing, the MEG signals were submitted to online and off-line noise reduction (Robinson, 1989). Prior to recording, individual skull/scalp landmarks were spatially coregistered (left and right preauricular points, $\mathrm{Cz}$, nasion, inion) using a Polhemus 3Space Fastrak system. The landmark locations in relation to sensor positions were derived on the basis of precise localization signals provided by 5 spatially distributed head coils with a fixed spatial relation to the landmarks. These landmarks were matched with the individual subjects' anatomical magnetic resonance (MR) scans. The T1-weighted MR images were scanned with a neuro-optimized GE Signa LX 1.5 T system (General Electric, Milwaukee, USA) using a quadrature head coil and a 3D- 


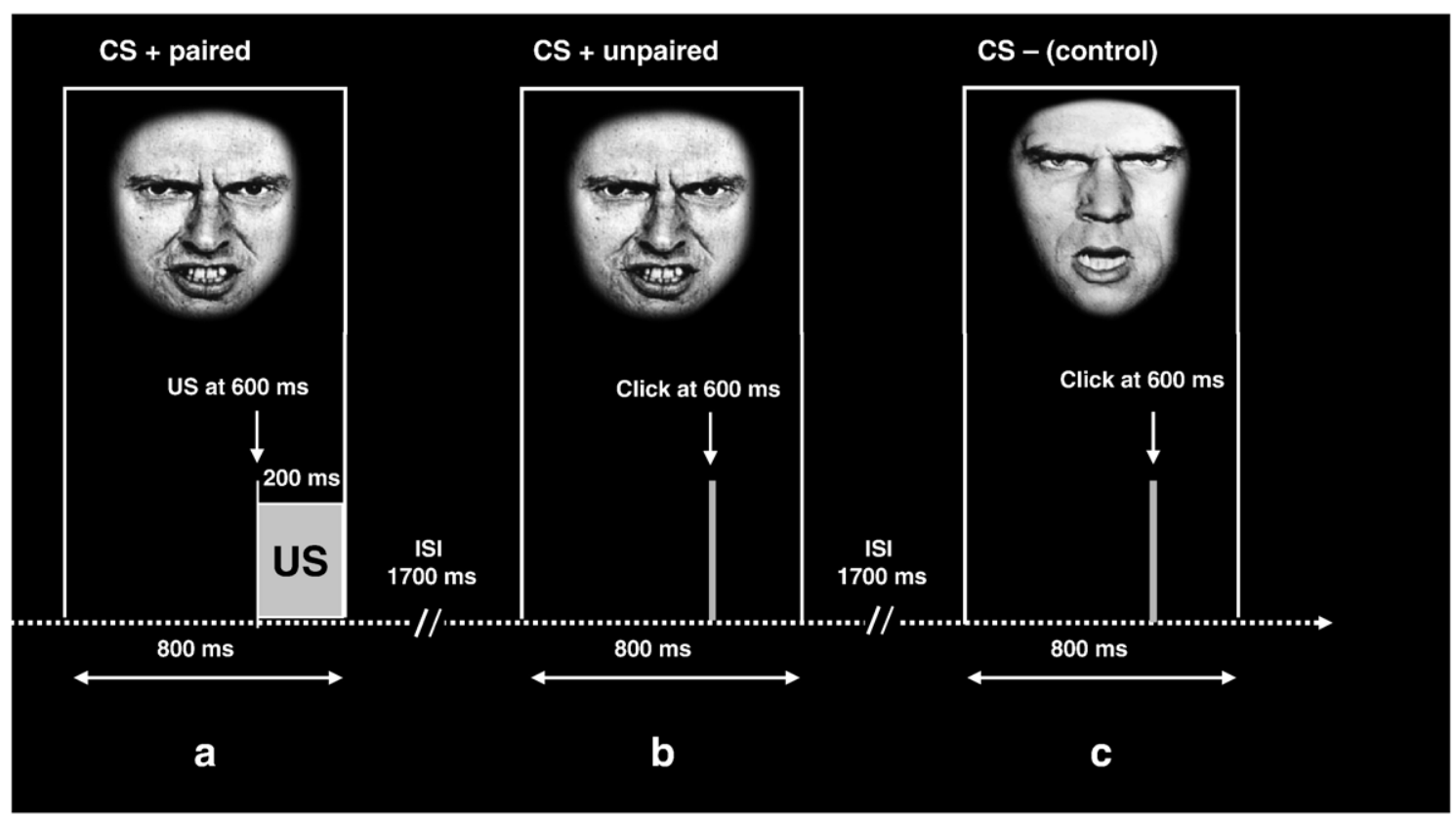

Fig. 1. Illustration of the experimental paradigm: a male angry face is presented persisting for $800 \mathrm{~ms}$. (a) One of the two face identities was conditioned with an aversive sound (white noise, $100 \mathrm{~dB}, 200-\mathrm{ms}$ duration) starting after $600 \mathrm{~ms}$ with a $50 \%$ probability and referred to as condition 'CS ${ }_{\text {paired }}$ '. (b) Alternatively, a single brief auditory click followed after presentation of this face; condition ' $\mathrm{CS}+{ }_{\text {unpaired' }}$. (c) A second face not previously paired with an aversive noise is shown, and an auditory click (as in b) is presented after $600 \mathrm{~ms}$. The sequence of the three trial types was randomized and balanced over the experiment. The association of the two faces with the conditioning (the aversive noise) was balanced over the subjects.

SPRG echo sequence, TR/TE/flip angle $=24 \mathrm{~ms} / 8 \mathrm{~ms} / 24^{\circ}$, spatial resolution $1 \mathrm{~mm} \times 1 \mathrm{~mm} \times 1.5 \mathrm{~mm}$, in-plane resolution: $256 \times$ 256, 124 slices, no gap).

To compute the grand average activity, the MR-related coordinate system for each subject was readjusted to the coordinate system of one subject whose anatomical MR scan was used for the grand average source analysis. By means of anatomical landmarks mentioned before the MRI scan was, in turn, spatially normalized into the standardized reference (Talairach and Tournoux, 1988), for the purpose of reporting MEG source localization coordinates.

Separate event-related fields (ERFs) were derived from the MEG epochs by selectively averaging over artefact-free epochs

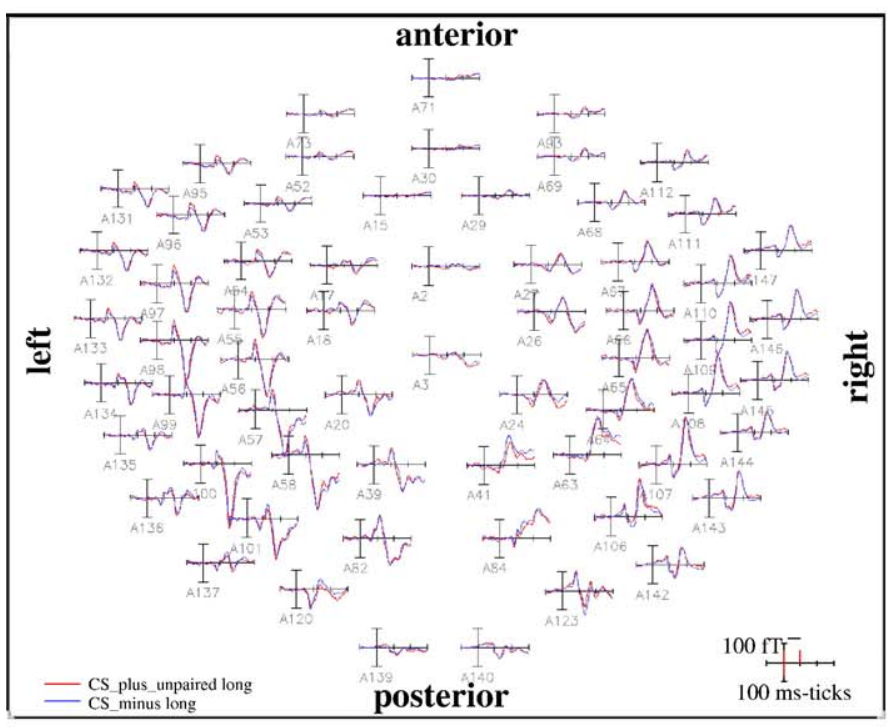

a

\section{Difference $\mathrm{CS}_{+}$- $\mathrm{CS}$ Average Fields (152 ms) ${ }^{-}$Inc.-5ft}

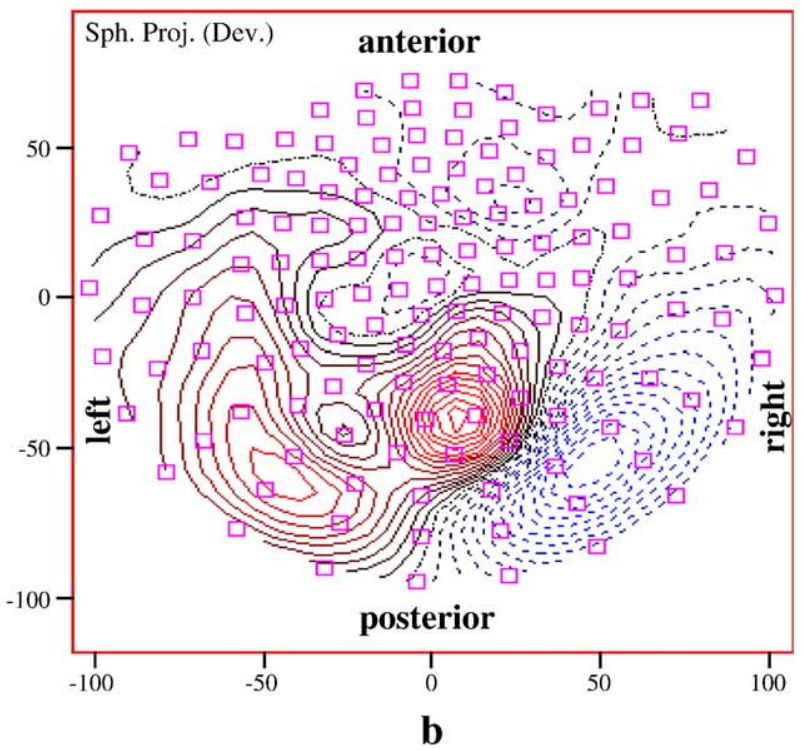

Fig. 2. (a) Distribution of grand average MEG waveforms over the head as observed after the onset of face presentation. Only every second sensor is included in this figure. Blue: $\mathrm{CS}-$ condition. Red: $\mathrm{CS}+_{\text {unpaired }}$ condition (i.e., only those sweeps where no aversive stimulus followed). (b) Topographical distribution of the grand average amplitude differences $\left(\mathrm{CS}_{+}{ }_{\text {unpaired }}-\mathrm{CS}-\right)$ at $152 \mathrm{~ms}$ after onset of face presentation. 

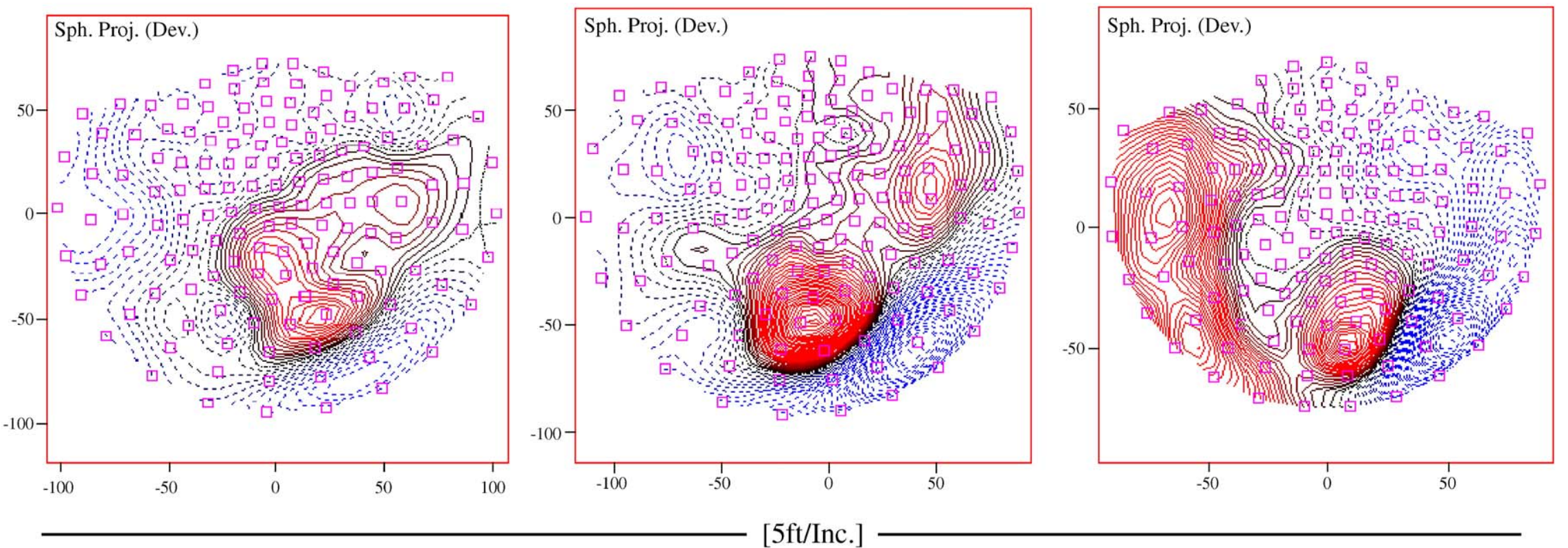

Fig. 3. Topographical distribution of the amplitude differences (CS+ $\left.{ }_{\text {unpaired }}-\mathrm{CS}-\right)$ at $152 \mathrm{~ms}$ after onset of face presentation for three representative single subjects. See Fig. 1 for the location of the sensors. 

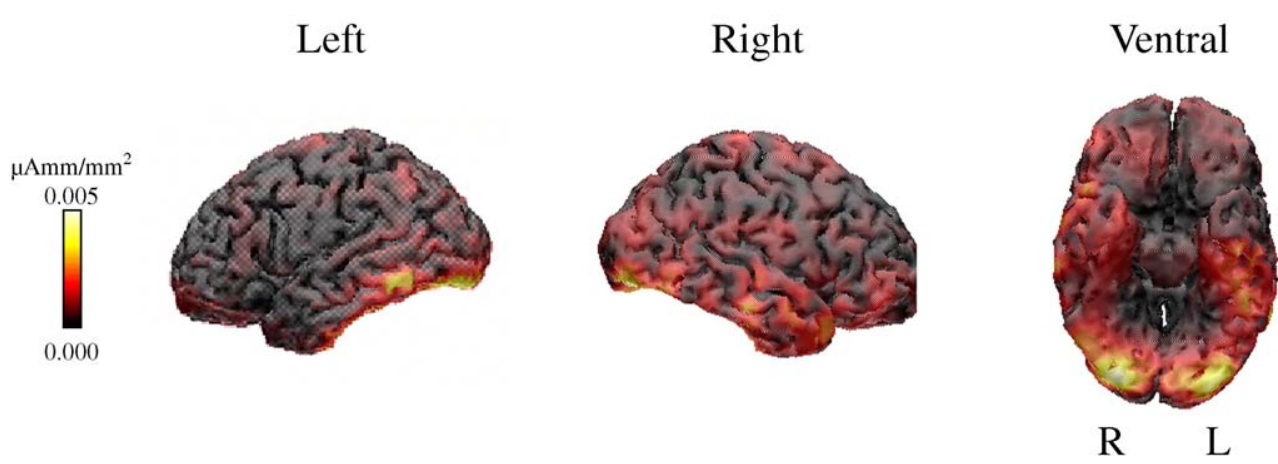

Fig. 4. Distributed source model for the grand average difference amplitude distribution shown in Fig. 1.

(artefact threshold $3 \mathrm{pT}$ peak-to-peak for the MEG) for each condition (i.e., $\mathrm{CS}-, \mathrm{CS}{ }_{\text {paired }}$ and $\mathrm{CS}+{ }_{\text {unpaired }}$ ) and for the face stimulus and the auditory stimulus. Epochs containing eye movement or blink artifacts were excluded based on an absolute amplitude artifact criterion $(100 \mu \mathrm{V})$ applied to both the horizontal and vertical EOG. The subsequent analysis was restricted to ERFs following the $\mathrm{CS}-$ and $\mathrm{CS}{ }_{\text {unpaired }}$ stimuli because, after the aversive stimulus ( $\mathrm{CS}+{ }_{\text {paired }}$ condition), a startle response occurred generating strong EOG artefacts. Individual subject's ERFs were recalculated for a standardized grid of 148 virtual magnetometer positions using a method previously proposed (Ilmoniemi, 1993) as implemented in the ASA program (A.N.T. Software, Enschede/ Holland). From these standardized data, the grand average fields (GAV) were derived. Also, these recalculated amplitude values were used for the subsequent statistical analysis. Deriving the GAV this way may temporally smear the resulting waveforms because the latencies of the specific components may vary over all subjects. However, we applied this procedure because it is consistent with the long standing tradition of analyzing event-related potentials. The amplitudes of the various components of the ERFs were statistically analyzed using an analysis of variance (ANOVA) for repeated measures with a varying number of factors depending on the kind of analysis. Greenhouse-Geisser correction for inhomogeneity of variance was used whenever a factor had more than two levels. Statistical significance was assumed if the likelihood $\mathrm{p}$ for a chance effect was below 0.05 . The amplitude values resulted from an average over a $12 \mathrm{~ms}$ ( 3 samples) interval centered around the latencies under consideration. They were referenced to the baseline derived as the average over the $100-\mathrm{ms}$ interval immediately preceding the onset of the respective stimulus. Further details regarding the factors included in the analyses are specified in the Results section. Grand average ERF waveforms were used to illustrate the overall ERF waveforms and topography in the relevant time windows as well as to perform representative source analyses for the group of subjects.

MEG source analysis was performed using the multimodal neuroimaging software (Curry, Version 4.0, Neuroscan Inc., 1999). First, a 3-D reconstruction of the head and cortical surface was created (boundary element model, BEM (Hamalainen and Sarvas, 1989) using the segmented anatomical MRI scan. Second, this model of the head and brain was used in conjunction with the observed MEG fields to compute a model of the distribution of current dipoles over the cortical surface (henceforth called a distributed source model) using the minimum norm least squares method (Fuchs et al., 1999). The BEM surface grid served as a predefined source compartment. Current densities were restricted to the cortex surface (excluding the cerebellum). The fit was optimized guided by two simultaneous criteria: first, to minimize the reconstruction error of the forward calculated amplitudes and, second, to minimize the cumulated squared source moments (L2norm). In order to counteract the tendency of this method to overemphasizing superficial source locations, a regularization procedure was applied that adds a weighted noise term to the total error term used for optimization (see Neurosoft, CURRY user guide, version 4.0, 1999, pp. 156-160 (Fuchs et al., 1999).

\section{EOG processing}

In order to estimate the potential influence of residual ocular movements (startle response) on the MEG results separate auditory click-related EOG waveforms were derived for each of the two conditions (i.e., $\mathrm{CS}-$ and $\mathrm{CS}+{ }_{\text {unpaired }}$ ) including all epochs not exceeding the artifact threshold of $\pm 100 \mu \mathrm{V}$.

\section{Suppression of contingent MEG activity}

Similar to the well-known contingent negative variant (CNV; Walter et al., 1964) which occurs in event-related potentials if a warning stimulus is (Walter et al., 1964) followed by a imperative stimulus, a continuous signal increase or decrease is observed in some of the ERF waveforms after the face presentation. This signal slope might even extend beyond the onset of the subsequent auditory stimulus thereby leading to a distortion of the ERF components elicited by this stimulus. Therefore, before analyzing these components, the total ERF, starting $100 \mathrm{~ms}$ before onset of face presentation was filtered with a phase corrected high pass filter with cut off frequencies $1 \mathrm{~Hz}$ (end of stop band) and $2 \mathrm{~Hz}$ (begin of pass band). In addition, we used a separate baseline for the auditory components in order to suppress potential residual contingent activity. Finally, we estimated the topographic amplitude distribution of the condition-related contingent activity by computing the amplitude differences $\left(\mathrm{CS}+{ }_{\text {unpaired }}-\mathrm{CS}-\right)$ before filtering for each sensor at $600 \mathrm{~ms}$ after onset of the face presentation (i.e., at the onset of the

Fig. 5. (a) Distribution of grand average MEG-waveforms (high pass filtered, see manuscript) over the head as observed after the onset of the auditory stimulus. Only every second sensor is included in this figure. Blue: CS- condition Red: $\mathrm{CS}^{+}{ }_{\text {unpaired }}$ condition. (b) Topographical distribution of the grand average amplitude (CS-) at $50 \mathrm{~ms}$ after onset of auditory stimulus. (c) Topographical distribution of the grand average amplitude differences $\left(\mathrm{CS}+{ }_{\text {unpaired }}-\mathrm{CS}-\right.$ ) at $180 \mathrm{~ms}$ after onset of auditory stimulus. (d) Same as panel c but at $220 \mathrm{~ms}$. 

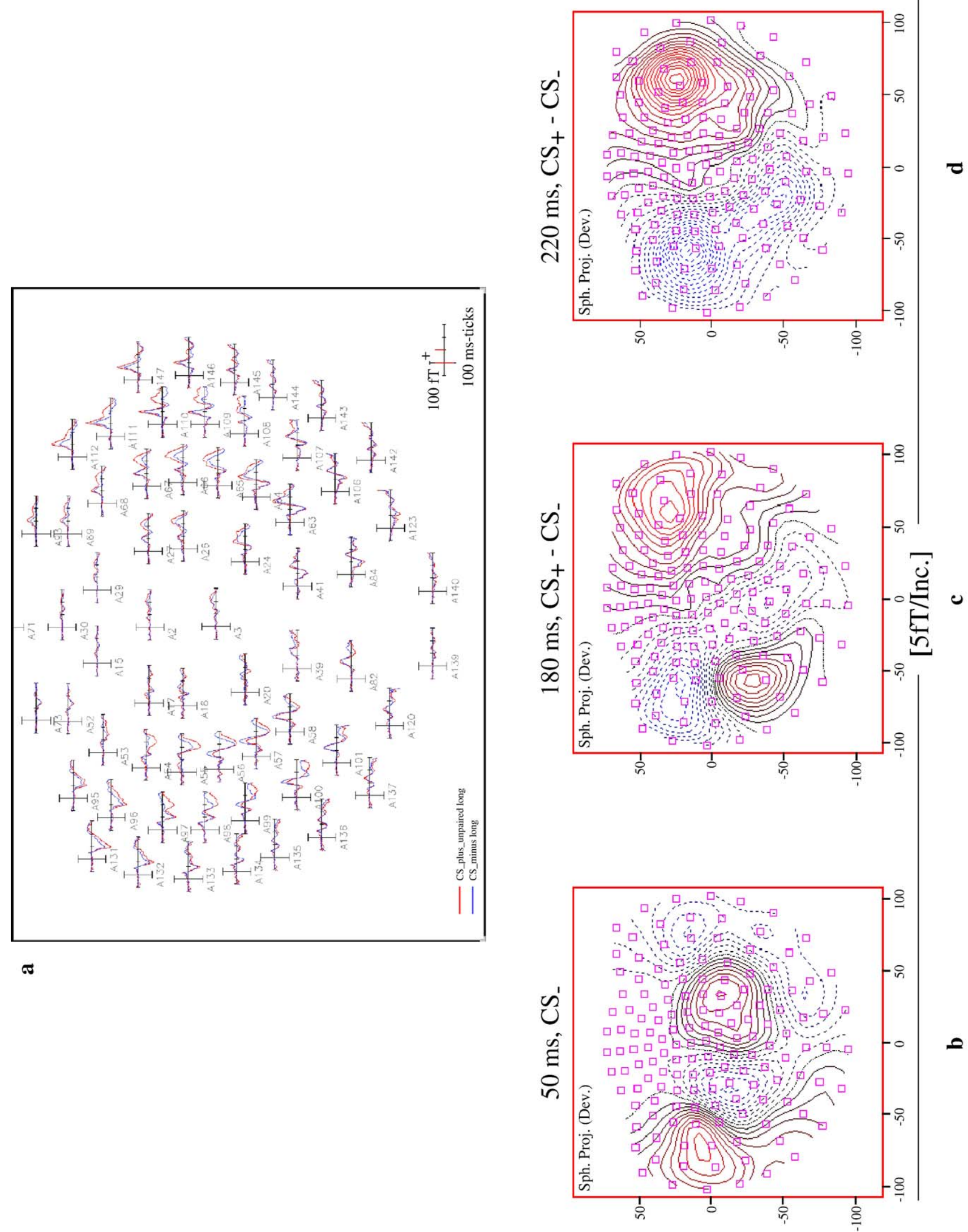
auditory stimulus) and plotted its topographic distribution. The resulting topographic distribution did not mimic any of the specific filtered difference components observed after presentation of the click, thus making unlikely any assumption of residual contingent activity to be its origin.

\section{Results}

\section{Behavioral measurements}

In a separate study, we obtained reaction times for subjects making push button responses to each acoustic stimulus to provide an operant measure of conditioning. Response time data, tested for a main effect of conditioning $\left(\mathrm{CS}+{ }_{\text {unpaired }}\right.$ versus $\mathrm{CS}-$ stimuli across the whole experiment), indicated significantly faster reaction times to $\mathrm{CS}+{ }_{\text {unpaired }}\left(\mathrm{RT}_{\text {mean }} 488 \mathrm{~ms}\right)$ stimuli compared to $\mathrm{CS}-\left(\mathrm{RT}_{\text {mean }}\right.$ $625 \mathrm{~ms}$ ) stimuli (one-way ANOVA, $F(1,10)=21.7, P=0.001$ ).

\section{MEG measurements}

\section{Face processing}

The observed grand average (GA) MEG signals exhibited multiple peaks which were most pronounced over occipito-parietal and occipital regions (see Fig. 2a). A first component showing a difference between $\mathrm{CS}{ }_{\text {unpaired }}$ and $\mathrm{CS}-$ arises at $80 \mathrm{~ms}$ and extends over a latency range up to $120 \mathrm{~ms}$ (according to the typical latency range of the ordinary visual P1 component observed in electrically evoked visual potentials). The differences were most pronounced over centro-occipital sensors at latencies of 100 to $110 \mathrm{~ms}$, with the absolute amplitudes larger under the $\mathrm{CS}+{ }_{\text {unpaired }}$ condition. However, even at sensors exhibiting the largest difference, this effect does not reach significance although there was a strong trend towards significance $(P>0.06$ with a two-way ANOVA with 'electrode site' (two levels) and 'condition' as factors).

The first statistically significant difference for the comparison of $\mathrm{CS}{ }_{\text {unpaired }}$ and $\mathrm{CS}-$ began at $130 \mathrm{~ms}$ and continued until $180 \mathrm{~ms}$ after stimulus onset with a peak at $150 \mathrm{~ms}$ (see Fig. 2a). This effect was most evident over right occipito-central sensors (see Fig. 2b) where the amplitudes were decreased under the $\mathrm{CS}+{ }_{\text {unpaired }}$ condition.

Representative single subject data illustrating this effect are shown in Fig. 3. By shifting a window of $12 \mathrm{~ms}$ width $(=3$ samples) over the waveforms, moving average amplitudes were calculated individually for all sites in both conditions. A three-way ANOVA with the factors 'electrode site' (two levels) and 'pole' (which means the positive or negative part of the dipole, i.e., two levels) and 'condition' showed a significant condition by pole interaction, over the latency range 134 to $158 \mathrm{~ms}$, peaking at 146 $\operatorname{ms}(F(1,9)=13,37 / P=0.005)$.

A current distribution source model was fitted to the grand average (GA) difference waveforms at 150-ms latency. As shown in Fig. 4, this analysis was consistent with a bilateral distribution of sources in the occipito-inferior cortex, more pronounced on the right side.

\section{Processing of auditory stimulus}

\section{Early component, primary auditory cortex}

After the acoustic click, a first component appeared in the GA waveform after about $40 \mathrm{~ms}$ extending up to $60 \mathrm{~ms}$ with a peak at $50 \mathrm{~ms}$, see Fig. 5a. The field, with a clear dipolar topography component, was located almost symmetrically in both hemispheres over mid temporal sensors, see Fig. 5b. This distribution is in line with an assumption of the primary auditory cortex as its likely origin. There was no condition-related statistically significant (two sensors, two-way ANOVA, $P>0.2$ for the condition main effect and for the condition* ${ }^{*}$ site interaction) modulation of these two components according to an ANOVA as outlined before.

\section{Later components}

A second component with peak latency at $130 \mathrm{~ms}$ with a complex topography exhibited a condition-related difference (CS+ versus $\mathrm{CS}-$ ) mainly over left centro-parietal sensors. However, at the 4 sensors showing the largest differences, a two-way ANOVA did not show a statistically validated modulation $(P>0.28$ for the condition main effect and for the condition*site interaction).

The first condition-related statistically significant differential effect occurred at a topographically focused component peaking at $180 \mathrm{~ms}$ (Fig. 5a) at sensors on the left fronto-temporal area. Correspondingly, the difference field (Fig. 5c) is located on the left temporal side exhibiting a focused dipolar topography reflecting the reduced absolute amplitudes under the $\mathrm{CS}^{+}$unpaired condition (seen in Fig. 5a). Representative single subject data are provided in Fig. 6. At the same latency, a less pronounced and less focused non-dipolar difference field was evident in a right fronto-temporal area. Statistically, a three-way ANOVA (as described before) analyzing 12-ms average amplitudes selected from the center of the difference on the left side indicated a non significant condition main effect (due to the positive and negative poles of the dipole canceling each other out in the analysis) and a significant condition*pole interaction $(F(1,9)=20.01 / P<0.002)$.

An additional two-way ANOVA for the amplitudes obtained at 4 sensors at the center of the negative pole of the dipolar field distribution resulted in a non significant condition main effect $(F(1,9)=0.002 / P>0.9)$ but a significant condition* site interaction $(F(3,27)=16.5 / P=0.001)$. A corresponding two-way ANOVA for the right hemisphere's difference amplitude showed a significant condition main effect $(F(1,9)=6.24 / P=0.034)$ but no significant condition*site interaction $(F(3,27)=0.09 / P>0.9)$. This can be explained as due to the fact that the topographically broad distribution of the right hemisphere difference field the relative amplitudes between the sensor remain constant whereas the absolute level changes with condition. Opposite to the condition specific modulation on the left side, on the right side, the differences reflected a topographical extension of a peak at 180 ms latency towards anterior regions. Taking together the different topographical field structure and modulation in the differences across the two hemispheres are suggestive of different processes. The differences on the right side presumably indicate the onset of the later bilateral process described below.

In order to localize the underlying generators, we performed a current distribution source analysis at 180-ms latency. As can be seen from Fig. 7, this analysis implicated primarily fronto-temporal regions extending to orbito-frontal structures. However, given the large distance between the orbito-frontal cortex and the MEG sensors and taking into account the poor sensitivity of the MEG for deep sources, this source localization should be interpreted with care - the signals are probably generated somewhere in the area, but the exact location cannot be specified with certainty.

A topographically broadly distributed bilateral peak (see Fig. $5 \mathrm{a}$ at sensors marked by the dashed line) starts at about $180 \mathrm{~ms}$ and continues to a latency of $270 \mathrm{~ms}$. The absolute amplitude 

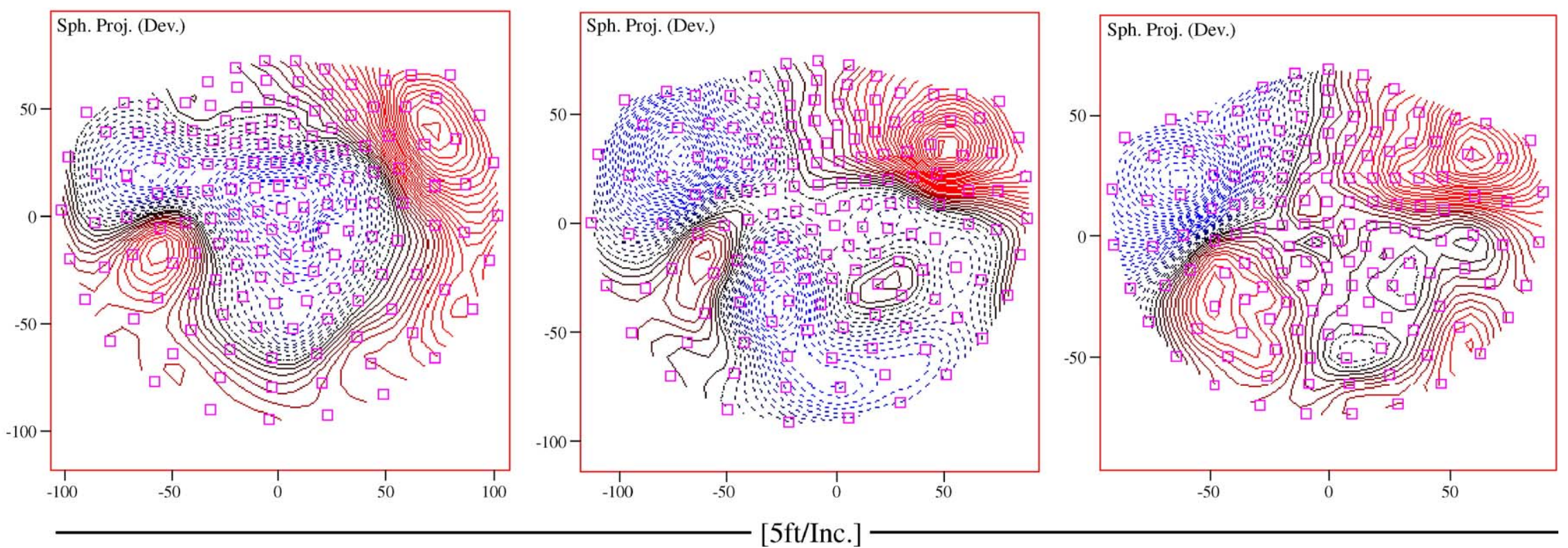

Fig. 6. Topographical distribution of the amplitude differences (CS+ ${ }_{\text {unpaired }}-\mathrm{CS}-$ ) at $180 \mathrm{~ms}$ after onset of auditory stimulus for three representative single subjects. See Fig. 1 for the location of the sensors. 

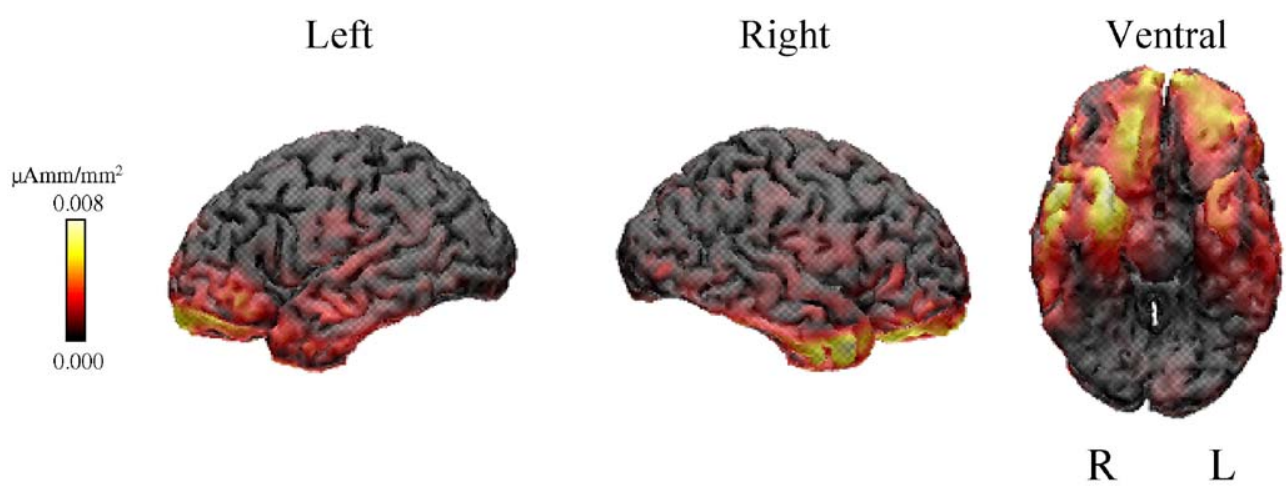

Fig. 7. Distributed source model for the grand average difference amplitude distribution at $180 \mathrm{~ms}$ (as shown in Fig. 4c).

of this component is increased under the $\mathrm{CS}+{ }_{\text {unpaired }}$ condition. The corresponding difference field is distributed bilaterally (Fig. 5d) with the center located over frontal and anterior temporal sensor sites. This difference reaches its maximum between 220 $\mathrm{ms}$ and $260 \mathrm{~ms}$ depending on the sensor site. A two-way ANOVA performed for the amplitudes at 3 sites out of the centers of the two poles yields a significant condition main effect with $F(1,9)=7.16 / P=0.025$ (condition*site interaction not significant, $F(2,18)=3.9, P>0.11)$ on the right side and as well a significant main effect $(F(1,9)=13.557 / P=0.005)$ and a nonsignificant condition* site interaction $(F(2,18)=0.39, P>0.69)$ on the left side. The source analysis of this component, see Fig. 8, again suggests fronto temporal regions to be involved in the underlying neural process.

\section{EOG measurements}

The vertical EOG (Fig. 9) showed an early component with onset around $70 \mathrm{~ms}$ and extending up to about $300 \mathrm{~ms}$. The condition-related difference waveform (i.e., $\mathrm{CS}+{ }_{\text {unpaired }}-\mathrm{CS}-$ ) was restricted to the latency range 70 to $170 \mathrm{~ms}$ indicating that eye movements that might have occurred did not differ between the two conditions before $70 \mathrm{~ms}$ and beyond $170 \mathrm{~ms}$. In the horizontal EOG (Fig. 9), no activity at all was observed.

\section{Discussion}

In this study, we show distinct modulations in visual and auditory-evoked MEG responses in the context of classical conditioning. These modulations are expressed, with different time courses, in sensory regions that correspond to the CS+ (visual) and UCS (auditory) stimuli. Specifically, conditioning is associated with an early modulation, peaking at $150 \mathrm{~ms}$, of visual-evoked responses to a conditioned face that precedes the typical face response seen at $170 \mathrm{~ms}$. Likewise, we observed modulations in responses to a probe auditory stimulus in a time window that incorporated an expected occurrence of an auditory UCS.

The early occipital modulations in response to the CS+ face (relative to the $\mathrm{CS}_{-}$) indicates that emotional learning is associated with experience-dependent changes in the temporal processing of a conditioned stimulus. In animal models of conditioning plastic changes within sensory cortex that processes the CS have been demonstrated as a consequence of emotional learning (Edeline and Weinberger, 1991; Edeline and Weinberger, 1993; Weinberger, 1993). Similar effects have been observed in human functional neuroimaging studies for processing of an auditory conditioned stimulus (Thiel et al., 2002). However, these studies do not speak to sensory dynamics of CS processing with conditioning. Thus, the time course of the modulations we describe indicates effects beyond primary sensory regions in so far as they are expressed at later time points than typical early sensory visual-evoked responses that occur at approximately $60-$ $80 \mathrm{~ms}$. Indeed, the time of maximal change in response to conditioned stimuli accords with a putative location within occipito-temporal cortex of the associated sources in extrastriate regions seen in the current data.

An early modulation (M100) in face response has been reported for neutral faces during a face categorization task which was stronger when a face was correctly perceived as a face than as a
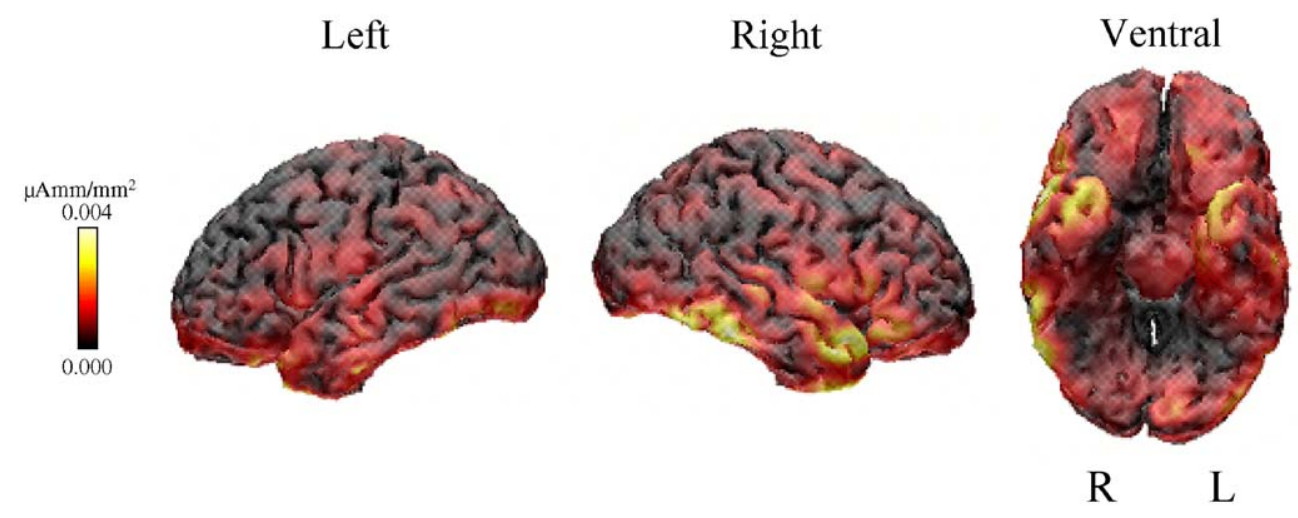

Fig. 8. Distributed source model for the grand average difference amplitude distribution at $220 \mathrm{~ms}$ (as shown in Fig. 4d). 
Vertical EOG
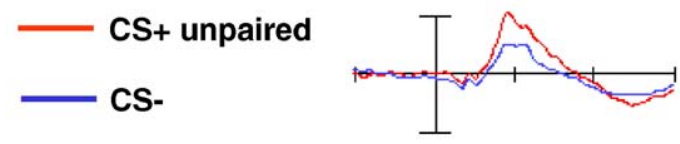

Difference

CS+ unpaired - Cs-

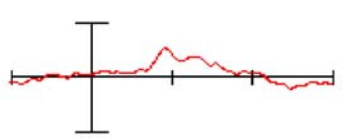

Horizontal EOG
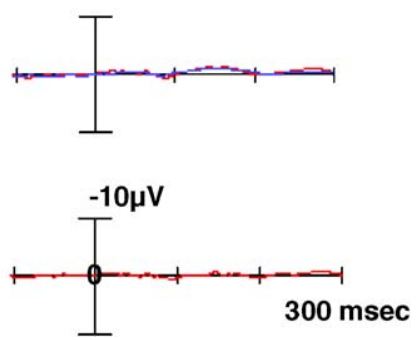

Fig. 9. EOG (grand average) averaged over all epochs which were included in the averaging of MEG activity related to the auditory clicks. The scale marked in the lower right applies to all four subfigures. A latency of $0 \mathrm{~ms}$ corresponds to the onset of the auditory click.

non-face(Liu et al., 2002). The modulations we report cannot be equated with these early responses in that our findings represent differential responses to face stimuli that emerge with conditioning to one of the faces under identical task requirements. More pertinent to the current data are previous studies that have examined the influence of emotion on electrophysiological responses to faces. The face-specific N170 component measured with ERPs is unaffected by emotional expression (Eimer et al., 2003). This lack of effect on the N170 contrasts with evidence that fearful faces elicit a fronto-central positivity at about $120 \mathrm{~ms}$ poststimulus presentation, followed by a broadly distributed sustained positivity beyond $250 \mathrm{~ms}$ post-stimulus (Eimer and Holmes, 2002). Using MEG, an enhancement of an M150 response to faces expressing happiness and disgust, relative to neutral expression, has also been reported (Lewis et al., 2003). Indeed short-latency differential responses in occipital regions, occurring at approximately $110 \mathrm{~ms}$ post-stimulus, have been reported to emotional compared to neutral faces (Halgren et al., 2000).

There have been few studies that have examined conditioningrelated effects on electrophysiological responses measured with EEG or MEG. An exception is a recent ERP study that reported differences between CS + and CS- as early as approximately 120 $\mathrm{ms}$ post-stimulus with a putative source in ventral extrastriate visual regions (Pizzagalli et al., 2003). Our MEG finding of an early modulation in MEG responses that peaks at approximately $150 \mathrm{~ms}$ accords with these ERP findings. The differential nature of the effects points to an emotional learning basis to the observed extrastriate modulations. One suggestion is that these modulations reflect re-entrant influences from amygdala that serve to enhance perceptual fluency to sensory events of value (Dolan, 2002). The time course of the differential responses, most marked at about 150 $\mathrm{ms}$, is in keeping with evidence that human amygdala itself responds to faces as early as $120 \mathrm{~ms}$ s (Halgren et al., 1994).

In auditory cortex we tested for modulation of auditory-evoked responses by presenting a click at the expected time of receipt of the auditory UCS. The issue here is whether expectation of an aversive UCS leads to modulation in sensory processing occurring with the same domain. Auditory cortex plasticity seen in previous animal and human studies has been reported to occur within the CS+ modality (Edeline et al., 1993; Weinberger, 1993; Morris et al., 1998b; Thiel et al., 2002). An amygdala-dependent auditory cortex plasticity expressed in the development of late $(500-1500 \mathrm{~ms})$ conditioned tone responses in auditory cortex to an auditory CS+ has also been reported (Quirk et al., 1997). Pizzagalli et al. reported modulation in auditory cortex, peaking at approximately $412 \mathrm{~ms} \mathrm{~s}$, following a CS+ prior to delivery of an auditory UCS (Pizzagalli et al., 2003). The modulation we observed is similar and would accord best with an attentional modulation in this region based upon expectancy of receipt of a UCS. The time of occurrence of these modulations (180 $\mathrm{ms}$ to $220 \mathrm{~ms}$ ), and their topography, again indicates influences beyond an early sensory modulation. Their relatively late onset and their fronto-temporal distribution are more consistent with modulations based upon engagement of higher cognitive processes such as anticipation and expectancy. Modulation in neural response with expectancy has been reported within prefrontal cortical regions (Critchley et al., 2001).

Given the differential fronto-temporal activation following the auditory click one could suspect that some mild eye movements not rejected by the artifact criterion might contribute to or even generate this component as these artefacts have a similar topographical distribution. However, throughout the latency range of this specific differential ERF component (starting at $180 \mathrm{~ms}$ ), the difference EOG stays at low amplitudes (see Fig. 9), thus discounting-though not fully ruling out-this interpretation.

Do the findings of early modulation with in sensory processing of a CS stimulus provide evidence for privileged processing of emotional stimuli (Dolan, 2002)? The early differential component is consistent with this proposal as it shows that emotional learning has selective effects on the time course of sensory processing of a $\mathrm{CS}+$. We have previously suggested an amygdala-driven emotional modulation of extrastriate cortex is likely to provide that underlying mechanism for the type of effect we observe in the present study (Morris et al., 1998a). Our findings fit a model that processing of the emotional value of a CS + stimulus by the amygdala provides a context for a modulatory signal to the relevant sensory cortex. This mechanistic account is supported by evidence for emotional modulation of the attentional blink for words an effect reversed by damage to the amygdale (Anderson and Phelps, 2001).

In conclusion, we show that emotional learning, as expressed in the context of classical conditioning, is associated with the emergence of a differential time course in processing a visual $\mathrm{CS}+$ relative to a $\mathrm{CS}-$ stimulus. Within the modality of the UCS, there is also modulation of sensory processing based upon expectancy of its occurrence. The data indicate that acquired emotional value is associated with differential time courses in processing predictive sensory stimuli as well as modulations within the sensory domain of the UCS itself.

\section{Acknowledgment}

This work was supported by the following grant: BMBF \#01GO0504 (CAI). 


\section{References}

Allison, T., Puce, A., Spencer, D.D., McCarthy, G., 1999. Electrophysiological studies of human face perception: I. Potentials generated in occipitotemporal cortex by face and non-face stimuli. Cereb. Cortex 9 , $415-430$.

Anderson, A.K., Phelps, E.A., 2001. Lesions of the human amygdala impair enhanced perception of emotionally salient events. Nature 411, 305-309.

Bentin, S., Allison, T., Puce, A., Perez, R., McCarthy, G., 1996. Electrophysiological studies of face perception in humans. J. Cogn. Neurosci. 8, 551-565.

Buchel, C., Dolan, R.J., 2000. Classical fear conditioning in functional neuroimaging. Curr. Opin. Neurobiol. 10, 219-223.

Chuang, S., Otsubo, H., Hwang, P., Orrison, W.M., Lewine, J.F., 1995. Pediatric magnetic source imaging. Neuroimaging Clin. N. Am. 5 (2), 289-303.

Critchley, H.D., Mathias, C.J., Dolan, R.J., 2001. Neural activity in the human brain relating to uncertainty and arousal during anticipation. Neuron 29, 537-545.

Critchley, H.D., Mathias, C.J., Dolan, R.J., 2002. Fear conditioning in humans: the influence of awareness and autonomic arousal on functional neuroanatomy. Neuron 33, 653-663.

Damasio, A.R., Damasio, H., Van Hoesen, G.W., 1982. Prosopagnosia: anatomical basis and behavioural mechanisms. Neurology 32, $331-341$.

Dolan, R.J., 2002. Emotion, cognition, and behavior. Science 298, $1191-1194$.

Dolan, R.J., Fletcher, P., Morris, J., Kapur, N., Deakin, J.F., Frith, C.D., 1996. Neural activation during covert processing of positive emotional facial expressions. NeuroImage 4, 194-200.

Edeline, J.M., Weinberger, N.M., 1991. Thalamic short-term plasticity in the auditory system: associative returning of receptive fields in the ventral medial geniculate body. Behav. Neurosci. 105, 618-639.

Edeline, J.M., Weinberger, N.M., 1993. Receptive field plasticity in the auditory cortex during frequency discrimination training: selective retuning independent of task difficulty. Behav. Neurosci. 107, 82-103.

Edeline, J.M., Pham, P., Weinberger, N.M., 1993. Rapid development of learning-induced receptive field plasticity in the auditory cortex. Behav. Neurosci. 107, 539-551.

Eimer, M., Holmes, A., 2002. An ERP study on the time course of emotional face processing. NeuroReport 13, 427-431.

Eimer, M., Holmes, A., McGlone, F.P., 2003. The role of spatial attention in the processing of facial expression: an ERP study of rapid brain responses to six basic emotions. Cogn. Affect. Behav. Neurosci. 3, 97-110.

Ekman, P., Friesen, W., 1982. Felt, false, and miserable smiles. J. Nonverbal Behav. 6, 238-252.

Fuchs, M., Wagner, M., Kohler, T., Wischmann, H.A., 1999. Linear and nonlinear current density reconstructions. J. Clin. Neurophysiol. 16, 267-295.

Halgren, E., Baudena, P., Heit, G., Clarke, J.M., Marinkovic, K., Chauvel, P., Clarke, M., 1994. Spatio-temporal stages in face and word processing: 2. Depth-recorded potentials in the human frontal and Rolandic cortices. J. Physiol. (Paris) 88, 51-80.

Halgren, E., Raij, T., Marinkovic, K., Jousmaki, V., Hari, R., 2000. Cognitive response profile of the human fusiform face area as determined by MEG. Cereb. Cortex 10, 69-81.

Hamalainen, M.S., Sarvas, J., 1989. Realistic conductivity geometry model of the human head for interpretation of neuromagnetic data. IEEE Trans. Biomed. Eng. 36, 165-171.

Haxby, J.V., Ungerleider, L.G., Horwitz, B., Maisog, J.M., Rapoport, S.I., Grady, C.L., 1996. Face encoding and recognition in the human brain. Proc. Natl. Acad. Sci. U. S. A. 93, 922-927.

Haxby, J.V., Petit, L., Ungerleider, L.G., Courtney, S.M., 2000. Distinguishing the functional roles of multiple regions in distributed neural systems for visual working memory. NeuroImage 11, 380-391.
Ilmoniemi, R.J., 1993. Models of source currents in the brain. Brain Topogr. 5, 331-336.

Jeffreys, D.A., 1989. A face-responsive potential recorded from the human scalp. Exp. Brain Res. 78, 193-202.

Kanwisher, N., McDermott, J., Chun, M.M., 1997. The fusiform face area: a module in human extrastriate cortex specialised for face perception. J. Neurosci. 17, 4302-4311.

Kawasaki, H., Kaufman, O., Damasio, H., Damasio, A.R., Granner, M., Bakken, H., Hori, T., Howard, M.A. III, Adolphs, R., 2001. Singleneuron responses to emotional visual stimuli recorded in human ventral prefrontal cortex. Nat. Neurosci. 4, 15-16.

LaBar, K.S., Gatenby, J.C., Gore, J.C., LeDoux, J.E., Phelps, E.A., 1998. Human amygdala activation during conditioned fear acquisition and extinction: a mixed-trial fMRI study. Neuron 20, 937-945.

Leahy, R.M., Mosher, J.C., Spencer, M.E., Huang, M.X., Lewine, J.D., 1998. A study of dipole localization accuracy for MEG and EEG using a human skull phantom. Electroencephalogr. Clin. Neurophysiol. 107 (2), $159-173$.

LeDoux, J.E., 1993. Emotional memory systems in the brain. Behav. Brain Res. 58, 69-79.

Lewis, S., Thoma, R.J., Lanoue, M.D., Miller, G.A., Heller, W., Edgar, C., Huang, M., Weisend, M.P., Irwin, J., Paulson, K., Canive, J.M., 2003. Visual processing of facial affect. NeuroReport 14, 1841-1845.

Liu, J., Harris, A., Kanwisher, N., 2002. Stages of processing in face perception: an MEG study. Nat. Neurosci. 5, 910-916.

Lu, S.T., Hamalainen, M.S., Hari, R., Ilmoniemi, R.J., Lounasmaa, O.V., Sams, M., Vilkman, V., 1991. Seeing faces activates three separate areas outside the occipital visual cortex in man. Neuroscience 43 , 287-290.

McCarthy, G., Puce, A., Belger, A., Allison, T., 1999. Electrophysiological studies of human face perception: II. Response properties of facespecific potentials generated in occipitotemporal cortex. Cereb. Cortex 9, 431-444.

Meadows, J.C., 1974. The anatomical basis of prosopagnosia. J. Neurol. Neurosurg. Psychiatry 37, 489-501.

Morris, J.S., Friston, K.J., Buchel, C., Frith, C.D., Young, A.W., Calder, A.J., Dolan, R.J., 1998a. A neuromodulatory role for the human amygdala in processing emotional facial expressions. Brain 121, $47-57$.

Morris, J.S., Friston, K.J., Dolan, R.J., 1998b. Experience-dependent modulation of tonotopic neural responses in human auditory cortex. Proc. R. Soc. Lond., B Biol. Sci. 265, 649-657.

Pizzagalli, D.A., Greischar, L.L., Davidson, R.J., 2003. Spatio-temporal dynamics of brain mechanisms in aversive classical conditioning: highdensity event-related potential and brain electrical tomography analyses. NeuroReport 41, 184-194.

Pourtois, G., deGelder, B., Vroomen, J., Rossion, B., Crommelinck, M., 2000. The time-course of intermodal binding between hearing and seeing affective information. NeuroReport 11, 1329-1333.

Quirk, G.J., Armony, J.L., LeDoux, J.E., 1997. Fear conditioning enhances different temporal components of tone-evoked spike trains in auditory cortex and lateral amygdala. Neuron 19, 613-624.

Robinson, S.E., 1989. Environmental noise cancellation for biomagnetic measurements. In: Williamsen, Hoke, M. (Eds.), Advances in Biomagnetism. Plenum Press, New York, pp. 721-724.

Sams, M., Hietanen, J.K., Hari, R., Ilmoniemi, R.J., Lounasmaa, O.V., 1997. Face-specific responses from the human inferior occipitotemporal cortex. Neuroscience 77, 49-55.

Sergent, J., Ohta, S., macDonald, B., Zuck, E., 1994. Segregated processing of facial identity and emotion in the human brain: a PET study. Vis. Cogn. 1, 349-369.

Talairach, J., Tournoux, P., 1988. Co-Planar Stereotaxic Atlas of the Human Brain. George Thieme Verlag, Stuttgart.

Thiel, C.M., Friston, K.J., Dolan, R.J., 2002. Cholinergic modulation of experience-dependent plasticity in human auditory cortex. Neuron 35, $567-574$. 
Walter, W.G., Cooper, R., Aldridge, V.J., Winter, M., 1964. Contingent negative variation. Nature 203, 360-380.

Watanabe, S., Kakigi, R., Puce, A., 2001. Occipitotemporal activity elicited by viewing eye movements: a magnetoencephalographic study. NeuroImage 13, 351-363.
Weinberger, N.M., 1993. Learning-induced changes of auditory receptive fields. Curr. Opin. Neurobiol. 3, 570-577.

Winston, J.S., O’Doherty, J., Dolan, R.J., 2003. Common and distinct neural responses during direct and incidental processing of multiple facial emotions. NeuroImage 20, 84-97. 